\title{
Noch schwankt der Boden: \\ Die katholische Presse in der Gegenwart (II)
}

\author{
von K. Rüdiger Durth
}

\section{Optimismus reicht nicht aus}

Zunehmend interessieren sich auch die publizistischen Fachzeitschriften für die konfessionelle Presse ${ }^{1}$, kehrt die Zukunft für die katholische Presse zurück ${ }^{2}$, sorgen die großangelegten Werbekampagnen etwa der Arbeitsgemeinschaft Katholischer Presse $(\mathrm{AKP})^{3}$ für innerkirchliche Aufmerksamkeit, so ist doch vor einem falschen Optimismus zu warnen, der leicht zu einem sanften Ruhekissen werden kann. Denn trotz aller Anstrengungen - etwa der Medien-Dienstleistungsgesellschaft (MDG) - und wohlgemeinten Festreden zum jährlichen „Tag der sozialen Kommunikationsmittel” sieht die Wirklichkeit an der Basis oft anders aus.

So heißt es beispielsweise im Jahresbericht 1978 des Zentralkomitees der deutschen Katholiken $(\mathrm{ZdK})^{\mathbf{4}}$ : „Größerer Aufmerksamkeit bedarf die Kontaktpflege zur Kirchenpresse. Es ist bisher nicht gelungen, alle Redaktionen persönlich kennenzulernen. Es müßte gelingen, die Redaktionen der Bistumspresse dazu zu bewegen, auf Veranstaltungen des ZdK durch eigene Redakteure vertreten zu sein.”

Im Klartext: Auf der langen Pressebank der Frühjahrs- und Herbstvollversammlungen des höchsten katholischen Laiengremiums findet man nur selten einen Redakteur der 22 Bistumszeitungen, ausgenommen die Kirchenzeitung des Erzbistums Köln und das „Ruhrwort”. Die anderen 20 Redaktionen verlassen sich offensichtlich auf die Katholische Nachrichtenagentur (KNA), obwohl gerade diese Vollversammlungen von knapp zweitägiger Dauer den Bistumsblatt-Redaktionen gute Möglichkeiten für eigene Schwerpunkte der Berichterstattung, der Interviews mit führenden Repräsentanten der katholischen Kirche und des Kontaktes mit Vertretern der säkularen Medien bieten.

K. Rüdiger Durth ist Pastor in der Evangelischen Kirche des Rheinlandes und hauptberuflicher Redakteur der „Bonner Rundschau”. 
Bleiben wir beim Zentralkomitee der deutschen Katholiken stehen, das selbst - keineswegs im Interesse der eigenen Sache - in den letzten Jahren in immer kürzerer Zeit seine Pressereferenten auswechselte und im Mai 1979 kaum in der Lage war, Mitglieder für seine Publizistik-Kommission zu finden. Einige der vom geschäftsführenden Ausschuß vorgeschlagenen ZdK-Mitglieder baten in der Vollversammlung um die Zuweisung in andere Kommissionen. Mit Recht fragte daraufhin Generalsekretär Dr. Friedrich Kronenberg, ob die Vollversammlung überhaupt eine solche Kommission noch wolle.

Die Kommission unter Vorsitz von Dr. Hermann Boventer gestand ihrerseits im bereits erwähnten Jahresbericht des ZdK ein, daß sie sich mehr mit Absichten beschäftige, als konkrete Ergebnisse vorlegen könne. Und das liest sich dann so:

„Es gelang der Kommission nicht, das seit langem auf der Tagesordnung stehende Thema 'Katholische Journalisten in der Tagespresse' ausführlicher anzugehen und zu einer breiteren Stellungnahme auszuformulieren. Es lagen zwar Erfahrungsberichte zu diesem Themenkomplex vor, die deutlich machten, daß die Kirche im Bereich der Tagespresse an Boden gewinnen muß und kann, sie reichten aber nicht aus, um daraus konkrete Forderungen und Vorschläge zu formulieren. Dies lag zum Teil auch darin begründet, daß einzelne Sitzungen (insgesamt vier in einem Jahr, Anm. des Verf.) sehr schlecht besucht waren und daß diejenigen fehlten, auf deren Sachwissen die Weiterberatung angewiesen war." 5

Diese nachlässige Behandlung publizistischer Belange an der Basis beziehungsweise im Alltag stellt keinesfalls einen Einzelfall dar und wird auch nicht durch den Einwand aufgehoben, man habe doch auch positive Arbeit geleistet. Im Blick auf die PublizistikKommission etwa der Verweis auf die Erklärung des ZdK zur Situation der Bistumspresse im gleichen Jahresbericht ${ }^{6}$. Entscheidender ist eben die kontinuierliche und an den alltäglichen Bedürfnissen der katholischen Publizistik orientierte Arbeit.

Die Wirklichkeit der Arbeit in den Redaktionen katholischer Zeitschriften, um ein weiteres Basis-Problem zu nennen, rechtfertigt ebenfalls keinen Optimismus. Die noch immer viel zu kleine Zahl hauptamtlicher Redakteure erlaubt es etwa den meisten Bistumsblättern kaum, einen Mitarbeiter für zwei Tage zur Teilnahme an einer Vollversammlung des $\mathrm{ZdK}$ freizustellen. Gleiches gilt für viele andere wichtige katholische Veranstaltungen.

Konkret heißt dies wiederum: Die ständig geforderte Verbesserung der inhaltlichen Qualität von Bistumspresse und anderen katholischen Zeitschriften läßt sich nur dann in die Tat umsetzen, wenn den Redakteuren dieser Zeitschriften auch die Möglichkeit eingeräumt wird, an den wichtigsten Veranstaltungen der Kirche selbst teilzunehmen und den Kontakt mit Kollegen von kirchlichen und säkularen Medien zu pflegen. Eigene Berichterstattung und persönliche Kontakte bilden für den kirchlichen Redakteur zwei Seiten dergleichen Medaille, nämlich besseren Journalismus.

Um hier einen Bewußtseinswandel herbeizuführen, wird es noch einer großen Überzeugungskraft bedürfen. Weniger aus finanziellen Gründen - obwohl diese in vielen Fällen auch eine Rolle spielen - als vielmehr aus Gründen der eingefahrenen Gleise. Optimismus allein reicht also nicht aus, um die zurückgekehrte Zukunft der katholischen Presse auszubauen und konsequent fortzuentwickeln. Die größte Hoffnung wird man auf den Nachwuchs setzen müssen - und wohl auch dürfen. Vorausgesetzt, daß 
die katholische Kirche ihre beispielhaften Ansätze in dieser Richtung tatkräftig fortentwickelt.

\section{Zehn Jahre Münchener Institut}

Im November 1979 feiert eine Journalistenschule ihr zehnjähriges Bestehen mit einem großen Fest im Ludwigshafener Hermann-Pesch-Haus, die inzwischen weit über die katholische Kirche als Träger hinaus Anerkennung gefunden hat: das Institut zur Förderung publizistischen Nachwuchses e.V. in München. Leider sind vorerst die Pläne gescheitert, diesem Institut unter der Leitung des Chefredakteurs der Zeitschrift „Stimmen der Zeit”, Dr. Wolfgang Seibel SJ, und des Geschäftsführers, Dr. Wilfried Schwedler, einen zeitgemäßeren Namen zu geben, etwa Görres-Institut. Namen aber sind nicht entscheidend, wohl aber die inhaltlichen Konzeptionen. Und dieses Institut zur Förderung publizistischen Nachwuchses hat in den letzten Jahren mehr für den katholischen Journalistennachwuchs getan als alle anderen Programmentwürfe und Festreden zusammengenommen.

Auf zwei Säulen ruht dieses Institut: Berufsbegleitende journalistische Ausbildung von Studenten aller Fachrichtungen und die Fort- und Weiterbildung von katholischen Journalisten, vor allem der Volontäre an katholischen Zeitschriften.

152 Stipendiaten wurden von 1970 bis einschließlich 1979 aufgenommen. Die Zahl der Bewerber war um ein Vielfaches höher und steigt von Jahr zu Jahr. Da das Institut weder die Qualität der Ausbildung gefährden noch ein ,journalistisches Proletariat" durch zuviele Journalisten fördern will, ist die Zahl der Stipendiaten pro Jahrgang inzwischen auf rund 15 begrenzt worden.

Viele Stipendiaten haben bereits führende Positionen innerhalb der Massenmedien inne, einer ist inzwischen Chefredakteur eines großen Bistumsblattes. Durch die große Zahl der Bewerber kann das Institut die besten auswählen (ohne deshalb elitär zu sein) und sie durch sogenannte Ferienakademien und vermittelte Praktikantenplätze in den Redaktionen von Presse, Funk und Fernsehen auf den Journalistenberuf nach erfolgreichem Abschluß des Studiums vorbereiten. Bei den säkularen Medien genießt das Münchener Institut längst einen ausgezeichneten Ruf, und viele Redaktionen geben bei Bewerbungen von Praktikanten unterschiedlicher journalistischer Schulen und Hochschulen denen des Münchener Instituts ganz bewußt den Vorzug. Nicht, weil die Studenten katholisch sind, sondern weil ihnen der Ruf vorauseilt, durch das Institut gut vorbereitet zu sein ${ }^{7}$.

In einer „Information für Bewerber und Interessenten” des 1969 im Auftrag der Deutschen Bischofskonferenz gegründeten und mit einem Jahresetat von rund 200.000 DM ausgestatteten Instituts heißt es:

„Aufgabe ist es, katholische Studentinnen und Studenten zu fördern, deren Absicht es ist, nach Studienabschluß einen publizistischen Beruf auszuüben. Das Studium soll jene Kenntnisse vermitteln, die Voraussetzung der journalistischen Arbeit in den verchiedenen Ressorts sind. Deshalb eignet sich jede Studienrichtung als Grundlage für einen journalistischen Beruf. Als Ergänzung des Studiums bietet das Förderungswerk seinen Stipendiaten eine praxisbezogene journalistische Schulung.

Eine Indoktrination der Stipendiaten liegt dem Institut fern; doch sollte sich jeder Interessent vor einer Bewerbung prüfen, ob er die Mindestvoraussetzung zur Aufnahme 
für seine Person bejahen kann: nicht eine bloß äußere Zugehörigkeit zur katholischen Kirche, sondern eine überzeugte christliche Grundhaltung, die später auch die eigene publizistische Arbeit prägen soll. Hinsichtlich der V.ahl ihres späteren Berufsplatzes als Journalist gehen die Stipendiaten keine Verpflichtung ein."

Das Förderungsangebot umfaßt die individuelle Beratung bei der Studienplanung, die Vermittlung berufsvorbereitender Praktika während der Semesterferien und die Veranstaltung von drei Ferienakademien (jeweils rund drei Wochen), die durch ihr theoretisches und praktisches Angebot sowie durch die Auswahl der Referenten bislang die meisten Volontäre einer Tageszeitung vor Neid erblassen lassen ${ }^{8}$.

\section{Das Volontärprogramm}

Die Volontärsausbildung für die Kirchenpresse (verantwortlich zeichnet Magnus Anton Dorn) wurde erst spät in das Programm des Münchener Instituts aufgenommen und in Zusammenarbeit mit der Medien-Dienstleistungsgesellschaft (MDG) und dèr Arbeitsgemeinschaft Katholischer Presse (AKP) entwickelt. Sie sieht vier vierzehntägige Ausbildungsseminare in einem Zeitraum von zwei Jahren vor. Die Volontäre wohnen während der Seminare zusammen - meist in einer katholischen Akademie - und haben so neben dem Ausbildungsprogramm die Möglichkeit des Erfahrungsaustausches untereinander. Im Mittelpunkt der Ausbildung stehen unter der Leitung namhafter Referenten theoretische Probleme des Journalismus und die Einübung in die wichtigsten journalistischen Stilformen.

Um zu zeigen, wie ein solcher Volontärkurs abläuft, wählen wir das Programm des Volontärkurses I, der vom 4. bis 16. Juni 1978 in der Katholischen Akademie St. Ulrich in Augsburg stattfand:

4. Juni Anreise bis zum Abendessen um 18,30 abends: Einführung und Filmvorführung über das Entstehen der Bistumszeitung „Kirche und Leben”.

5. Juni Richard Krön: Journalistische Gesprächsformen - ganztägig -

6. Juı Karl W. Mekiska: Die Nachricht - ganztägig-

7. Juni ganztägig: Nachricht

abends: Referat von Wilhelm Schätzler über „Die Organisation kirchlicher Medienarbeit"

8. Juni Peter Hans Reimann: Presserecht in der journalistischen Praxis - ganztägig-

9. Juni Norbert Denkel: Das Foto in der Zeitung - ganztägig-

10. Juni Norbert Denkel: Foto-Layout (Übungen zur Zeitungsgestaltung)

12. Juni Jens-Peter Eichmeier: Das Interview - ganztägig -

13. Juni Jens-P. Eichmeier: Pressekonferenz mit Finanzdirektor Helmut Weber über den Diözesanhaushalt von Augsburg - ganztägig -

14. Juni Heinz Schweden: Bericht-Reportage - ganztägig-

abends: Raimund Brehm: „Probleme marktorientierter Arbeit in christlichen Verlagen" 
15. Juni Heinz Schweden: Bericht-Reportage - ganztägig-

16. Juni Heinz Schweden: Besprechung der Übungsarbeiten

Neben diesen Volontärkursen - die Bistumszeitungen haben sich verpflichtet, ihre Volontäre daran teilnehmen zu lassen - veranstaltet das Institut Fortbildungsseminare für kirchliche Journalisten, zum Beispiel „Interpretation und Präsentation kirchlicher Haushaltspläne” vom 30. November bis 2. Dezember 1978 in Würzburg, ,Nachrichtenverarbeitung” vom 31. Januar bis 2. Februar 1978 in Ludwigshafen, „Ne'le Technik bei der Zeitungsherstellung" vom 6. bis 8. Februar 1979 in Stuttgart.

Auch diese Weiterbildungsmöglichkeiten sind dringend auszubauen, wobei das Münchener Institut längst an die Grenzen seiner Kapazität angelangt ist (zwei hauptamtliche Studienleiter, eine Sekretärin - welches Institut kommt mit diesem Personal aus?). Nur durch diese Selbsthilfe wird es der katholischen Publizistik gelingen, langfristig über eine größere Anzahl qualifizierter jüngerer (!) Journalisten zu verfügen.

Die Katholische Nachrichten-Agentur ermöglicht ihren Volontären darüber hinaus auch ein Praktikum bei einer Tageszeitung und/oder einer Rundfunkanstalt.

Die Medien-Dienstleistungsgesellschaft bemüht sich ebenfalls, den Redakteuren von Kirchenzeitungen Hilfestellungen für die inhaltliche Gestaltung zu geben. Das neueste Produkt: „Leser-Aktionen für die Kirchenpresse.”.

Die 50 Vorschläge und Ideen richten sich in erster Linie an die Redaktionen mit Vorschlägen, „wie die Wirkung der Kirchenzeitung in der Öffentlichkeit verstärkt werden könnte. Wie man Außenstehende auf die Kirche und ihre Presse in sympathischer Weise aufmerksam macht. Und wie man die Identifikation der vorhandenen Leser mit ihrer Zeitung festigt." 10

Bei diesen Vorschlägen handelt es sich um ganz praktische Tips für Geschichten, Themen und Aktionen, die sich eigentlich von selbst verstehen, aber in der Praxis gerade von den Kirchenzeitungen immer wieder vernachlässigt werden.

\section{Die bunte Palette}

Katholische Kirchenpresse - das sind nicht nur die 22 Bistumszeitungen" oder die Ordensblätter ${ }^{12}$, sondern auch Sonntagszeitungen, Jugendzeitschriften, Verbandsblätter und theologische Fachzeitschriften. Eine bunte Palette von periodischen Zeitschriften mit zum Teil erheblichen Auflagen.

Noch immer ist es nicht möglich, die ganze Farbenpracht dieser Palette zu malen, weil es an den notwendigen umfassenden Untersuchungen mangelt. Die wichtigsten Zeitschriften sind der Arbeitsgemeinschaft Katholischer Presse (AKP) ${ }^{13}$ angeschlossen, die auch eine genaue Statistik über die Auflagenentwicklung führt. Doch viele kleine Zeitschriften, deren Grenze oft zum Traktat nicht mehr genau festzulegen ist, fehlen. Dabei handelt es sich um periodische Publikationen von Ordensgemeinschaften, kleineren Verbänden, Missionsgesellschaften oder kirchlichen Werken.

Erinnert sei - um ein paar wenige Beispiele zu nennen - an die Zeitschrift der katholischen Akademie Klausenhof bei Bocholt, die vor allem über Jugendprobleme berichtet, an die Informationsschriften des Bundes Deutscher Katholischer Jugend. Aber auch an zeitlich begrenzte Publikationen, die meist mit Aktionen junger Katholiken verbun- 
den sind wie die Freiburger Katholikentagszeitschrift „Hoffnung” oder die Zeitschrift „Horizonte”, die achtmal mit einer Auflage von je 100.000 Exemplaren von der Arbeitsgemeinschaft Evangelischer Jugend und dem Bund Deutscher Katholischer Jugend zur 5 . Welthandelskonferenz der Vereinten Nationen im Mai 1979 herausgegeben wurde, um die deutsche Öffentlichkeit intensiver als bislang auf die Probleme der Entwicklungspolitik aufmerksam zu machen.

\section{Die Sonntagspresse}

Unter Sonntagspresse werden die Zeitschriften verstanden, die wöchentlich erscheinen, aber nicht an eine Diözese gebunden sind. Oft wenden sie sich an ganz bestimmte Zielgruppen, etwa die Familie. Die bekannteste - und zugleich umstrittenste Zeitschrift in dieser Gattung ist die „neue Bildpost”, die katholische Themen bildzeitungsgemä $\beta$ an den Mann beziehungsweise die Frau bringen will. Mit knalligen Schlagzeilen, oft genauso überzogen wie im Vorbild „Bild”, und politischer Einseitigkeit macht diese Wochenzeitung immer wieder auf sich aufmerksam.

Die „neue bildpost” ist an vielen Zeitungskiosken erhältlich, was wesentlich zu ihrem Bekanntheitsgrad beiträgt, und liegt in den Kirchen aus. Immer wieder aber kommt es vor, daß Bischöfe oder Pfarrer das Auslegen dieser Zeitschrift in den Kirchen verbieten oder nicht gern sehen. Daß die „neue bildpost” mit einer Auflage von gegenwärtig 227.000 Exemplaren nicht zu den besten Zeitschriften der katholischen Kirche zählt, darf nicht verschwiegen werden. Sie gilt übrigens auch nicht als offizielle Zeitschrift und mußte in den letzten Jahren zum Teil erhebliche Einbußen hinnehmen. Mit 377.000 Exemplaren erreichte sie Ende 1964 ihren Höchststand und fiel seit 1970 ständig unter die 300.000-Grenze. Der Abwärtstrend hält weiter an.

Insgesamt fiel die Auflage der Sonntagspresse von 919.000 Exemplaren im besten Jahr, Ende 1964, auf 578.000 Exemplare Ende 1978. Damit erreichte sie wieder den Stand von 1955, wo allerdings die "neue bildpost” nur mit 70.000 Exemplaren zu Buche schlug. Die stärksten Einbrüche in der Auflagenentwicklung mußte die „Christliche Familie" hinnehmen, die von 239.000 Exemplaren Ende 1962 auf jetzt 113.000 Exemplare Ende 1978 absackte. Das „Liboriusblatt” büßte von 1966 bis 1978 fast 100.000 Abonnenten ein: von 223.000 auf 162.000 .

Die Sonntagspresse $=61 \% .000$ Exemplare Gesamtauflage (Ende 1978)

\begin{tabular}{lllr}
\hline Altöttinger Liebfrauenbote & Altötting & wöchentlich & 40.000 \\
Bayerisches Sonntagsblatt & München & wöchentlich & 37.000 \\
Christ in der Gegenwalt & Freiburg & wöchentlich & 38.000 \\
Die Christliche Familie & Essen & wöchentlich & 113.000 \\
Liboriusblatt & Hamm & wöchentlich & 162.000 \\
neue bildpost & Schmallenberg & wöchentlich & 227.000
\end{tabular}

\section{Die Standes- und Verbandspresse}

Mit über zwei Millionen Exemplaren Auflage nimmt die Standes- und Verbandspresse einen wichtigen Platz innerhalb der katholischen Publizistik ein. Da diese Zeitschriften meist von den Mitgliedern der betreffenden Verbände bezogen werden und in der Regel der Preis im Mitgliedsbeitrag enthalten ist, halten sich die Auflagen- 
schwankungen dieser Zeitschriften in Grenzen. Da sie sich in erster Linie an die Mitglieder wenden, kommt ihnen die Funktion der innerverbandlichen Kommunikation zu, die jedoch angesichts der Bedeutung der Verbände keineswegs gering eingeschätzt werden darf.

$\begin{array}{lllr}\text { Auftrag } & \text { Bonn } & \text { zweimonatlich } & 5.000 \\ \text { Bauen und Siedeln } & \text { Köln } & \text { vierteljährlich } & 5.000 \\ \text { Bonifatiusblatt } & \text { Paderborn } & \text { vierteljährlich } & 510.000 \\ \text { Christ im Alter } & \text { Köln } & \text { monatlich } & 38.000 \\ \text { Der Kath. Mesner } & \text { Augsburg } & \text { monatlich } & 4.000 \\ \text { Die Christliche Frau } & \text { Köln } & \text { zweimonatlich } & 1.000 \\ \text { Die Mitarbeiterin } & \text { Düsseldorf } & \text { zweimonatlich } & 9.000 \\ \text { Frau im Leben } & \text { Augsburg } & \text { monatlich } & 339.000 \\ \text { Frau und Mutter } & \text { Düsseldorf } & \text { monatlich } & 589.000 \\ \text { Frau und Beruf } & \text { Düsseldorf } & \text { monatlich } & 11.000 \\ \text { Gemeinsame Aktion } & \text { Köln } & \text { 11 Ausgaben p. J. } & 5.000 \\ \text { Gemeinsame Zeitung } & \text { Köln } & \text { monatlich } & 171.000 \\ \text { Idee und Tat } & \text { Köln } & \text { vierteljährlich } & 18.000 \\ \text { Klerusblatt } & \text { München } & \text { monatlich } & 5.000 \\ \text { Kolpingblatt } & \text { Köln } & \text { monatlich } & 169.000 \\ \text { Königsteiner Rufe } & \text { Königstein } & \text { zweimonatlich } & 23.000 \\ \text { Lebendiges Zeugnis } & \text { Paderborn } & \text { vierteljährlich } & 8.000 \\ \text { Monika } & \text { Donauwörth } & \text { monatlich } & 82.000 \\ \text { Neue Mitte } & \text { Essen } & \text { zweimonatlich } & 14.000 \\ \text { Pax-Korrespondenz } & \text { Köln } & \text { zweimonatlich } & 18.000 \\ \text { Prisma der Frau } & \text { Köln } & \text { zweimonatlich } & 30.000 \\ \text { Ruf ins Volk } & \text { Hamm } & \text { monatlich } & 10.000 \\ \text { Unser Dienst } & \text { Köln } & 10 \text { Ausgaben p. J. } & 3.000 \\ \text { Weggefährte } & \text { Hamm } & \text { alle acht Wochen } & 8.000 \\ & & & \end{array}$

Stand: Ende Januar 1978

\section{Bunt darfes sein}

Bunt darf sie sein - die katholische Magazinpresse, die ebenso wie die Sonntagspresse die Aufgabe hat, „zu einer christlichen Lebensgestaltung in unserer Zeit durch zeitgemäße Bildung, Lebenshilfe und Unterhaltung aus shristlicher Verantwortung beizutragen"14. Die Unterhaltung kommt bei den Zeitschriften der Magazinpresse nicht zu kurz, zum Teil versteht sie sich sogar als Alternative zu den herkömmlichen Illustrierten, wie etwa das Augsburger "Weltbild”. Man kann es auch so formulieren: Die Magazinpresse will in erster Linie Lebenshilfe leisten, indem sie sich von vornherein auch in der Regelan ganz bestimmte Leserschichten wendet.

Die wichtigsten Titel:

- Weltbild. Erscheint alle vierzehn Tage mit farbigen Reportagen aus Gesellschaft, Kirche und dem alltäglichen Leben. Dazu ein komplettes TV-Programm. 
So sieht das Winfried-Werk Augsburg als Herausgeber von „Weltbild" seine Zeitschrift: „Weltbild-Leser wissen mehr, weil Weltbild keine Illustrierte unter vielen ist. Weltbild sagt ihnen, was sich hinter den Kulissen von Gesellschaft und Politik tut. Weltbild forscht nach den Hintergründen in praktischen Reportagen und kritischen Interviews. Weltbild zeigt Ihnen die Welt im Bild, wie sie wirklich ist - ungeschmin... ehrlich, aber auch faszinierend schön."

Obwohl Weltbild zu den wenigen großen katholischen Zeitschriften zählt, die an den Zeitungskiosken, in Lesemappen und an den Schriftenständen in den Kirchen erhältlich sind, kämpft die Redaktion mit dem Leserschwund.

„Weltbild" erfreute sich in den letżten Jahren durchaus bischöflichen Wohlwollens, das sich auch in erheblichen finanziellen Absicherungen ausdrückte. Ende 1968 verfügte die Zeitschrift über eine verkaufte Auflage von 703.000 Exemplaren, nachdem "Feuerreiter" und „Mann in der Zeit” in ihr aufgegangen waren. Ende 1949 hatte der „Feuerreiter" eine verkaufte Auflage von 244.000 Exemplaren, „Mann in der Zeit” von 242.000 Auflage, die Ende 1960 gar auf 673.000 Exemplare hochschnellte. Inzwischen sackte „Weltbild” auf 441.000 Exemplare ab (Ende 1978).

- Leben und Erziehen. Diese im Aachener Einhard-Verlag erscheinende Monatszeitschrift versteht sich als Magazin für Erziehungs- und Bildungsfragen und will zugleich Orientierungshilfen zur Lebensgestaltung der Familie geben. „Leben und Erziehen”, oft als Beilage für andere Zeitungen verwandt, hat eine kontinuierliche Aufwärtsentwicklung zu verzeichnen. Betrug Ende 1972 die verkaufte Auflage 107.000 Exemplare, so kletterte sie auf 664.000 Exemplare Ende 1978 bei einem Höhepunkt von 694.000 Exemplaren verkaufter Auflage Ende 1976.

- Erdkreis. Diese mit viel Bildern künstlerisch gestaltete Monatszeitschrift, die im Würzburger Echter-Verlag erscheint, konnte in den letzten Jahren ihre Auflage bei 21.000 Exemplaren halten.

- Kontraste. Kontrastreich hingegen die Auflagenentwicklung von „Kontraste”, einer Vierteljahreszeitschrift im Herder-Verlag Freiburg mit meist einem Schwerpunktthema. Die Auflage stieg von 18.000 Exemplare Ende 1970 auf 40.000 Exemplare Ende 1976, um zwei Jahre später auf die Hälfte zusammenzuschrumpfen.

- Zenit. Das monatliche Seniorenmagazin will diesem Leserkreis aktuelle Tips für die Lebensgestaltung geben und verzeichnet eine verkaufte Auflage von 36.000 Exemplaren Ende 1978. Das Magazin erscheint im Augsburger Hall-Verlag.

Die Gesamtauflage der Magazin-Presse betrug Ende 1978 1,182 Millionen verkaufter Exemplare. Hier die Verkaufsauflage Ende 1978 im Überblick:

\begin{tabular}{lllr}
\hline Erdkreis & Würzburg & monatlich & 21.000 \\
Kontraste & Freiburg & vierteljährlich & 20.000 \\
Leben und Erziehen & Aachen & monatlich & 664.000 \\
Weltbild & Augsburg & vierzehntäglich & 441.000 \\
Zenit & Augsburg & monatlich & 36.000
\end{tabular}




\section{Die vergessene Jugend}

Die katholische Jugendpresse spielt gegenwärtig innerhalb der Überlegungen einer zeitgemäßen christlichen Presse eine große Rolle. Dabei geht es zum einen um Möglichkeiten, Jugendliche stärker für die katholische Jugendpresse zu interessieren (die freilich keineswegs mit kommerziellen Zeitschriften wie „Bravo” oder „Freizeit-Revue” konkurrieren können), zum anderen aber auch darum, die Jugend als Abonnenten für Bistumszeitungen und andere periodische Zeitschriften zu gewinnen, da diese alle die Überalterung ihrer Leser beklagen. Kinder- und Jugendseiten gelten neuerdings als "Allheilmittel", was freilich bezweifelt werden darf.

Wie wichtig es ist, daß sich die kirchliche Publizistik intensiv um die Jugend kümmert, hat die Bundesvorsitzende des Bundes Deutscher Katholischer Jugend (BDKJ), Maria Koppernagel, auf der 29. Jahresversammlung der Arbeitsgemeinschaft Katholische Presse im Oktober 1978 in Augsburg deutlich gemacht:

"Was den für kirchliches Verhalten besonders signifikanten sonntäglichen Kirchgang
betrifft, so fand in den Jahren zwischen 1963 und 1976 bei der katholischen Jugend
ein starker Rückgang statt. Die Teilnahme der 16 bis 29jährigen sank von 52 auf
21 Prozent. Wenn 1976 der Tiefpunkt von 19 Prozent überschritten wurde, so sollte
man nicht voreilig auf eine Tendenzwende in diesem Verhaltersbereich schließen,
denn langfristig gesehen setzt sich ein protestantisches Kirchgangsverhalten durch, das
ab und zu sonntags zum Kirchgang bewegt." 15

Der abschließende Appell der BDKJ-Vorsitzenden an die Kirchenpresse verdient Beachtung: „So wichtig eigene Jugendseiten auch sein mögen, schieben Sie die Jugendlichen nicht auf einige Seiten Ihrer Publikationen ab; greifen Sie im Hauptteil Themen auf, die für Jugendliche und Erwachsene gleichermaßen interessant sind; lassen Sie öfter einmal Jugendliche zu den sie bedrängenden Fragen zu Wort kommen." 16

Die Jugend, die durch Schule, Jugendgruppen, Fernsehen und Verhalten der Erwachsenen immer mehr eigene Normen sucht und traditioneller Frömmigkeit und Kirchlichkeit skeptisch gegenübersteht, stellt an eine eigene katholische Presse hohe Anforderungen, will diese auch wirklich gelesen werden. Auf diesem Gebiet steht die katholische Publizistik - die evangelische nicht weniger - erst am Anfang.

Immerhin erreichen die in der AKP zusammengeschlossenen katholischen Jugendzeitschriften noch eine Auflage von rund zwei Millionen Exemplaren. Diese hohe Zahl wird aber nicht zuletzt dadurch erreicht, daß die Spitzenreiter wie „Sternsinger” zum Teil kostenlos verteilt werden.

\section{Die wichigsten katholischen Jugendzeitschriften:}

- Sternsinger. Eine Zeitschrift für Kinder im Alter von 6 bis 12 Jahren. Sie erscheint viermal im Jahr als Missions- und viermal als Diasporaausgabe.

- Freund der Kinder: Diese Monatszeitschrift aus dem Donauwörther Auer-Verlag wendet sich an die 6 bis 10jährigen und bringt Geschichten und Sachbeiträge aus allen Lebensbereichen, Rätsel und Bastelanweisungen.

- Junge Zeit. Dieses Monatsmagazin aus München ist gedacht für junge Leute zwischen 14 und 19 Jahren. Neben Informationen nimmt der Freizeitbereich einen breiten Raum ein. 
-17. Die Steyler Missionsgesellschaft gibt diese Zeitschrift für junge Christen „um 17” heraus, die Stellung zu Lebens- und Glaubensfragen beziehen.

- Spatz. Diese bunte Monatszeitschrift aus dem Düsseldorfer Klens-Verlag zählt sicher zu dem Besten, was es an Zeitschriften für das Kindergarten- und Grundschulalter gibt. Das Deutsche Jugendschriftenwerk empfiehlt diese Zeitschrift mit Recht.

Weite Welt. Diese Monatszeitschrift wendet sich an Schülerinnen und Schüler zwischen 8 und 13 Jahren. Jedes Heft enthält ein vierfarbiges Poster und wird ebenfalls von der Steyler-Missionsgesellschaft herausgegeben.

Top. wendet sich als Monatsmagazin an junge Christen zwischen 10 und 14 Jahren und erscheint im Nürnberger Sailer-Verlag.

Hier ein Überblick auf die Verkaufsauflage Ende 1978:

\begin{tabular}{lllr}
\hline Freund der Kinder & Donauwörth & monatlich & 18.000 \\
Junge Zeit & München & monatlich & 46.000 \\
17 & Nettetal & monatlich & 9.000 \\
Spatz & Düsseldorf & monatlich & 86.000 \\
Sternsinger/Mission & Aachen & vierteljährlich & 900.000 \\
Sternsinger/Diaspora & Paderborn & vierteljährlich & 810.000 \\
Top & Nürnberg & monatlich & 21.000 \\
Weite Welt & Nettetal & monatlich & 181.000
\end{tabular}

Kommunikation für den Experten

Klein an Zahl, aber keineswegs in der Bedeutung zu unterschätzen sind die Kulturund Fachzeitschriften innerhalb der katholischen Kirche. Sie bieten aktuelle Informationen aus dem jeweiligen Bereich, für den sie erscheinen, und Hintergrundberichte. In der Regel wendet sich diese Zeitschriftengruppe an Multiplikatoren. Nicht berücksichtigt sind die theologischen Zeitschriften im strengen Sinn.

Caritas. Diese Zweimonatszeitschrift versteht sich als Zentralorgan des Deutschen Caritas-Verbandes und informiert über die Caritasarbeit sowie die Caritaswissenschaft.

- Die Anregung aus St. Augustin erscheint monatlich und bietet neben Predigtskizzen, theologisch-wissenschaftlichen Beiträgen auch Stellungnahmen zu religiösen Zeitfragen.

- Film-Dienst bespricht vierzehntäglich alle neuen Filme im Kino und Fernsehen und berichtet über Entwicklungen im Filmbereich. Ziel dieser Zeitschrift ist es, den Dschungel der Filmindustrie etwas zu lichten und gute Filme zu empfehlen.

- Geist und Leben aus dem Würzburger Echter-Verlag versteht sich als Zeitschrift für spirituelle Theologie und Praxis. Sie erscheint alle zwei Monate.

- Herder-Korrespondenz. Diese Monatszeitschrift aus dem Herder-Verlag Freiburg zählt ebenso wie die „Stimmen der Zeit” zu den bekanntesten katholischen Zeitschriften im geistigen Leben und wendet sich an die Gebildeten im Lande. Die Zeitschrift für Gesellschaft und Religion bringt Analysen, Interviews, Dokumente, Meinungen, Zeitberichte und Tagungsberichte. 
- Mann in der Kirche ist als zweimonatiges Werkheft für die Männerseelsorge gedacht. Herausgegeben vom Augsburger Winfried-Werk bietet es mit theologischen und gesellschaftspolitischen Beiträgen Orientierungshilfen.

- Pastoralblatt. Diese Zeitschrift mit Informationen, Beratung und Anregungen für die Seelsorger erscheint im Kölner Bachem-Verlag für die Diözesen Aachen, Berlin, Essen, Köln und Osnabrück.

- Predica Verbum steht als Monatszeitschrift aus dem Donauwörther Auer-Verlag im Dienst der Glaubensverkündigung mit Predigtvorschlägen und Besprechung religiöser Literatur.

- Schule und Mission versteht sich als Handreichung für Lehrer zur missionarischen Bewußtseinsbildung, die vom Päpstlichen Missionswerk Aachen viermal im Jahr herausgegeben wird.

- Stimmen der Zeit wendet sich als Monatszeitschrift der Jesuiten vor allem Themen aus den Bereichen Religion, Gesellschaft, Politik, Naturwissenschaft und Literatur zu.

- ut omnes unum erscheint zweimonatlich beim Paderborner Winfriedbund „im Dienst der Einheit der Christen”. Es bietet Glaubensinformationen, Mitteilungen über Ökumenismus und Ostkirche.

- Die Mitarbeiterin erscheint zweimonatlich im Düsseldorfer Klens-Verlag als Werkheft für Frauenbildung und Frauenseelsorge.

Übersicht über die verkaufie Auflage Ende 1978 der Kultur- und Fachzeitschriften:

\begin{tabular}{llll}
\hline Caritas & Freiburg & monatlich & 2.000 \\
Christlicher Osten & Würzburg & zweimonatlich & 7.000 \\
Die Anregung & St. Augustin & monatlich & 2.000 \\
Filmdienst & Köln & vierzehntäglich & 3.000 \\
Geist und Leben & Würzburg & zweimonatlich & 4.000 \\
Herder-Korrespondenz & Freiburg & monatlich & 9.000 \\
Mann in der Kirche & Augsburg & zweimonatlich & 5.000 \\
Pastoralblatt & Köln & monatlich & 5.000 \\
Praedica Verbum & Donauwörth & monatlich & 2.000 \\
Stimmen der Zeit & Freiburg & monatlich & 5.000 \\
ut omnes unum & Paderborn & zweimonatlich & 1.000 \\
\end{tabular}

\section{Schwankender Boden}

Vergleicht man einmal die Auflagenentwicklung der Bistumszeitungen seit 1949, so büßten diese Zeitungen innerhalb von dreißig Jahren 8,1 Prozent ihrer Auflage ein. Bei näherem Hinsehen aber ergibt sich ein noch dramatischeres Bild:

Die Bistumszeitungen erreichten mit 2,396 Millionen Exemplaren Gesamtauflage im Jahr 1963 ihren Höhepunkt. Die Auflagensteigerung betrug in dem Zeitraum 1949 bis 1963 rund 402.000 Exemplare oder 29,2 Prozent. Der Auflagenverlust in den Jahren 1963 bis 1978 betrug 563.000 Exemplare, was 23,5 Prozent entspricht. 
An einigen Beispielen aus der Bistumspresse soll dies näher erläutert werden: Das „Ruhrwort” der Diözese Essen erreichte 1960, im ersten Jahr, eine Auflage von 147.000 Exemplaren, die bereits nach einem Jahr um 11.000 Exemplare geschwunden war. Die Abwärtsentwicklung hielt konsequent an. 1972 wurde erstmals die 100.000-Grenze erreicht. Ende 1977 wurden noch 82.000 Exemplare, Ende 1978 noch 77.000 Exemplare verkauft. Die Kirchenzeitung des Erzbistums Köln hatte 1949 eine Auflage von 218.000 Exemplaren, erreichte Ende 1958 mit 239.000 Exemplaren ihren Höhepunkt und zählt heute noch 151.000 verkaufte Exemplare. Die Bistumszeitung Würzburg hat 1978 wieder den Stand von 1949 mit 79.000 Exemplaren erreicht, mit 89.000 Exemplaren erreichte sie 1964 ihren höchsten Stand ${ }^{17}$.

Für Ende 1978 ergab sich für die katholische Presse, sofern sie der AKP angeschlossen ist, folgende Auflagenzahl ${ }^{18}$ :

Bistumspresse
Sonntagspresse
Magazinpresse
Standes- und Verbandspresse
Ordens- und Missionspresse
Jugendzeitschriften
Kultur- und Fachzeitschriften
Sonstige

Gesamtauflage
1.830.000 Exemplare
617.000 Exemplare
1.182.000 Exemplare
2.075.000 Exemplare
2.551.000 Exemplare
2.070.000 Exemplare
45.000 Exemplare
34.000 Exemplare

10.404.000 Exemplare

Vergleicht man die Auflagenentwicklung der Bistumspresse vom 4. Quartal 1977 zum 4. Quartal 1978, also im letzten Jahr, so ergibt sich ein Auflagenschund von einem Prozent oder 18.801 Exemplare. Gegenüber dem 4. Quartal 1976 zum 4. Quartal 1977 ein leichtes Abbremsen des Schwundes, nämlich um 0,3 Prozent. Bedenkt man aber, daß gerade im Jahr 1978 erhebliche Anstrengungen unternommen wurden, um die Bistumspresse stärker im Bewußtsein der katholischen Bevölkerung zu verankern, so muß dieser Auflagenschwund dennoch bedenklich stimmen ${ }^{19}$.

Immerhin konnten im vergangenen Jahr gegenüber 1977 einige Bistumszeitungen an Auflage gewinnen, so die Kirchenzeitung Berlin 86 Abonnenten (0,6 Prozent), die Bistumszeitung Eichstätt 21 Abonnenten (0,1 Prozent). Die größte Auflagensteigerung konnten die Bistumszeitungen Rottenburg (plus $\dot{4}, 9$ Prozent gleich 5.638 Abonnenten) und Regensburg (plus 2,2 Prozent, gleich 1.946 Abonnenten) verzeichnen, wobei die Bistumszeitung Rottenburg im Jahr davor drei Prozent Auflage verloren hatte. Die stärksten Verluste mußten im vergangenen Jahr die Bistumszeitungen Würzburg (minus 9,5 Prozent), Essen (minus 5,6 Prozent) und Hildesheim (minus 5,9 Prozent) hinnehmen ${ }^{20}$.

Die Sonntagspresse verlor 1978 0,3 Prozent (gleich 1.507 Exemplare) Auflage gegenüber dem tiefen Sturz von 1977 von 6,2 Prozent nit dem Spitzenverlierer Liboriusblatt (14,8 Prozent Verlust). Die Magazinpresse büßte 1978 2,5 Prozent Auflage ein (1977 1,1 Prozent) ${ }^{21}$. 


\section{Anmerkungen}

1 Raimund Brehm: Katholische Presse - „eine Presse für den Menschen”, ZV + ZV, 44-45/ 1978, S. 1785 ff; ders.: Die konfessionelle Presse: David oder Goliath im Pressemarkt?, ZV + ZV 17/1979, S. 667 ff; K. Rüdiger Durth: Der unbekannte Riese, in: „Journalist” 12/1977; ders.: „Die Krise ist überwunden”, in: „Journalist” 1/1979, S. 26 ff; ders.: „Auf dem Rückzug aus der Freiheit?" CS 12. Jg. (1979), S. 41 ff.

${ }^{2}$ K. Rüdiger Durth: Die Zukunft kehrt zurück: Die katholische Presse in der Gegenwart, Teil I, CS 12. Jg. (1979), S. $13 \mathrm{ff}$.

${ }^{3}$ Mitteilungen der Arbeitsgemeinschaft Katholische Presse e.V. Nr. 5 vom 30. Dezember 1978, S. 6.

4 Hektografiertes Manuskript, S. 23.

5 Ebd., S. 6.

6 Ebd.

7 Die Zahlenangaben beruhen auf Mitteilungen des Instituts gegenüber dem Vf, der selbst seit vielen Jahren Praktikanten des Instituts betreut und immer wieder aufs Neue über die journalist ische Begabung und Leistung der Stipendiaten erfreut ist. Einen guten Einblick in die „Ferienakademien” des Instituts gibt Gerhard Eberts: Reporter - nicht nur des Schreibens wegen, in: „Weltbild”, Nr. 10/1978.

$8 \mathrm{Vgl}$. Informationsblatt des Inștituts.

${ }^{9}$ MDG-Service, Bd. 2 „Leser-Aktionen für die Kirchenpresse”, 1979.

10 Ebd., S. 3.

$11 \mathrm{Vgl} . \mathrm{CS} 12 . \mathrm{Jg}$. (1979), S. $13 \mathrm{ff}$.

12 Ebd.

13 Die Angaben über Zeitschriften und Auflagenhöhen im Folgenden verdankt der Vf. der AKP, deren Geschäftsführer Bruno Geuter, sehr bemüht war, die Untersuchung über die Situation der katholischen Presse in der Gegenwart nach besten Kräften zu unterstützen.

14 Formuliert auf einer Redakteurskonferenz der AKP am 7. Juni 1967 auf Schloß Hirschberg in der Oberpfalz.

is Maria Koppernagel, Die religiöse Situation Jugendlicher - Möglichkeiten und Ansätze einer auf Kommunikation angelegten jugendarbeit. Referat auf der 29. Jahresversammlung der AKP im Oktober 1978 in Augsburg, hektogratiertes Manuskript, S. 4.

16 Ebd., S. 10.

17 Angaben laut AKP.

18 Ebd.

19 Ebd.

20 Ebd.

21 Ebd. 


\section{SUMMARY}

In the past decide the Catholic Church in Germany has made every eftort to solve the crisis of the Catholic Press. After the closure of the weekly "Publik", Catholic Media planners directed their attention to the Diocesian weeklies and publications such as "Rheinischer Merkur" (weekly) and "Weltbild" (bi-monthly), and to the strengthening of the Catholic News Agency (KNA) and improving training. This was necessary on account of the decrease in circulation and the gap between the media and the Church. One of the most important developments in Catholic Communications work has been the establishment of a Media Service Company (Medien-Dienstleistungsgesellschatt. MDG) in Munich in 1975. This was part of the communications emergency planning at the Synod of the German Dioceses in 1972. The association of the Catholic Press AKP - also helped towards a revival. New and improved cooperation among the diocesan weeklies - important instruments of dialogue within the Church - was developed. The author, in describing these developments, does not confine himself to publications, but also refers to press officers of the diocese and to publications from religious communities and missionary societies which have an important role in the press today. He feels that the crisis of the Catholic Press has been overcome, but that a strenuous effort is needed to implant this same press firmly into the German secular communications media.

(Part III of this contribution will be published in a later edition.)

\section{RÉSUMÉ}

Au cours de la dernière décennie, l'Eglise catholique fit des efforts considérables pour la presse catholique en crise. Après la suppression de l'hebdommadaire «Publik», la politique de mass media de l'Eglise consécra au renforcement des journaux épiscopaux, à la préservation du «Rheinischer Merkur» et du «Weltbild», de même que d'autres journaux et objets d'édition, au développement de l'agence de presse catholique (KNA) de même que l'encouragement de la relève journalistique. Ceci fut nécessaire parce que l'attachement à l'Eglise et avec lui le nombre d'exemplaires baissaient. Parmi lesprincipales nouveautés sur le marchè des mass media catholiques on compte aussi la sociéte de services des mass media (MDG) - fondée en 1975-à Munich comme partie du "programme publicistique express" et qui fut décidee en 1972 lors du synodecommun des évêchés allemands. La communauté de travail de la presse catholique (AKP) fait aussi partie des aides à l'intérieur de la publicistique catholique. A la suite d'effforts s'établit une collaboration plus étroite àl'intérieur des journaux épiscopaux qui, depuis des années, sont en train de changer fondamentalement. Ils prennent une part importante au dialogue à l'intérieur de l'Eglise. L'auteur expose les développements et ambitions avec le conflit d'opinions. En cela, il ne se limite pas seulement aux journaux épiscopaux, mais il aborde aussi les bureaux de presse des évêchés, de même que la presse des ordres et des missions qui est également une partie intégrante importante de la presse catholique actuelle. Il est persuadé que la crise proprement dite de la presse catholique est actuellement surmontée. De gros efforts sont assurément encore nécessaires pour assurer à la presse catholique la place qui lui revient à l'intérieur du paysage des mass media allemands.

(L'exposition détaillée est poursuivie dans une troisième partie.) 


\section{RESUMEN}

En el último decenio la Iglesia católica alemana hizo notables esfuerzos en pro de la prensa católica en crisis. Despues de la supresion del semanario «Publik», la politicaeclesial para medios de comunicación trato de robustecer la prensa diocesana, de asegurer al periodico "Rheinischer Merkur" y a la revista "Weltbild», así como a otras revistas y editoras, ampliando al mismo tiempo la Agencia Noticioșa Católica (KNA) y promocionando las vocaciones periodísticas. Todo ello fué necesario porque desminuyó el vínculo eclesial y con ello el número de suscriptores. Entre las más importantes novedades en el mercado de los medios de comunicación católicos se encuentra la fundación en 1975 de la MDG, una empresa de servicios para la comunicación social creada en Munich en el marco del "Programa Publicistico de Urgencia», aprobado en 1972 por el Sinodo Conjunto de las Diócesis Alemanas. También el Equipo de trabajo Prensa Católica (AKP) es uno de los puntales del publicismo católico en Alemania. Como consecuencia de estos esfuerzos surgió una estrecha colaboración entre los periódicos diocesanos, que se encuentran en evolución desde hace años. Esta prensa participa de forma importante en el diálogo interno eclesial. El autor presenta el desarrollo y aspiraciones, jonto con el antagonismos de las opiniones. No se limita a la prensa diocesana: se ocupa también de las oficinas de prensa episcopales, asi como de la prensa misionera y de las órdenes religiosas, que es parte importante de la prensa católica actual. Está convencido de que fué superada ya la crisis de lia prensal calólica. Sin embargo son necesarios todavia grandes esfuerzos para asegurar a la prensa católica el puesto que le corresponde en el panorama publicístico alemán.

(La exhaustiva exposición continuará en una tercera parte.) 